\title{
Efficacy of arsenic trioxide drug-eluting stents in the treatment of coronary heart disease
}

\author{
SHASHA ZHANG, YUPING ZHANG, SHICHUAN LI and ZHIFENG LI \\ Department of Cardiology, Yongchuan Hospital, Chongqing Medical University, Chongqing 402160, P.R. China
}

Received September 2, 2015; Accepted September 27, 2016

DOI: $10.3892 /$ etm.2017.4106

\begin{abstract}
The aim of the current study was to evaluate the safety and clinical efficacy of arsenic trioxide drug-eluting (AVI) stents, manufactured in China, for the treatment of coronary heart disease (CHD). Between January and August 2014, 40 patients with CHD admitted to Yongchuan Hospital with implanted AVI stents alone were selected. A one-year clinical follow-up was completed and one year postoperative coronary angiography was reviewed. Major adverse cardiovascular events (MACE), recurrent angina, stent restenosis and stent thrombosis cases were detected. All 40 patients with CHD completed the one-year clinical follow-up, as well as the one-year postoperative coronary angiography. The follow-up results indicated that the MACE rate was $15.0 \%(6 / 40)$, the target lesion revascularization rate was $15.0 \%(6 / 40)$, the angina recurrence rate was $32.5 \%$ (13/40), the in-stent restenosis rate was $20.0 \%(8 / 40)$ and the stent thrombosis rate was zero. There were no cases of cardiac death or nonfatal myocardial infarction. The incidence of restenosis was higher following implantation of the AVI stent and the safety and clinical efficacy were worse than expected.
\end{abstract}

\section{Introduction}

Coronary heart disease (CHD) is a common disease and usually occurs following the build up of plaque inside the coronary arteries that supply oxygen-rich blood to the heart muscle. This build-up can induce angiostenosis or angiemphraxis of the coronary arteries, reducing the flow of oxygen-rich blood to the heart, which may result in myocardial necrosis (1). Percutaneous coronary intervention (PCI) is an important therapeutic method of treating CHD (2). The primary factor leading to restenosis after PCI is smooth muscle cell proliferation and intimal migration

Correspondence to: Dr Zhifeng Li, Department of Cardiology, Yongchuan Hospital, Chongqing Medical University, 439 Xuanhua Road, Yongchuan, Chongqing 402160, P.R. China

E-mail: zhifenglidoc@163.com

Key words: arsenic trioxide, drug-eluting stent, percutaneous coronary intervention following local injury to the coronary artery $(3,4)$. It has been demonstrated that stents coated with anticancer drugs may significantly inhibit the proliferation of smooth muscle cells following PCI and reduce the restenosis rate (5). A number of studies have demonstrated that, as an anti-cancer agent, arsenic trioxide $\left(\mathrm{As}_{2} \mathrm{O}_{3}\right)$ is able to treat leukemia and other malignant tumors by inducing the apoptosis of tumor cells (6-12). The mechanism by which arsenic trioxide prevents restenosis is through attenuating smooth muscle cell proliferation by inhibiting the $\mathrm{G} 1$ and $\mathrm{S}$ phases of the cell cycle, as well as promoting apoptosis (13-15). Based on the aforementioned theory, novel domestically produced arsenic trioxide drug-eluting stents (AVI) have been developed, which have received a registration certificate issued by the State Food and Drug Administration (Beijing, China). Currently, AVI (Beijing Amsinomed Medical Device Co., Ltd., Beijing, China) is marketed for use in PCI, however, due to the lack of post-marketing clinical data, its safety and clinical efficacy are yet to be confirmed. The current study is a re-evaluation of prospective clinical studies with a monotherapy group on the basis of clinical registration, aimed at investigating the safety and clinical efficacy regarding the application of AVI stents in patients with coronary heart disease (CHD).

\section{Patients and methods}

Selection of cases. A total of 40 patients with CHD who underwent AVI implantation between January and August 2014 were selected from the cardiology department at Yongchuan Hospital of Chongqing Medical University (Chongqing, China). The present study consisted of 35 men and 5 women with a mean age of $63.4 \pm 11.6$ years. Patients were included in the study if they were $\geq 18$ years old, had symptomatic ischemic heart disease or presented with myocardial ischemia, in whom the stenosis diameter of at least one lesion was $\geq 70 \%$ and were suitable for implantation of AVI alone. Patients were excluded if they were pregnant, lactating or planning to become pregnant $\leq 1$ year following PCI, had an unusual susceptibility of bleeding or were diagnosed with coagulation disorders, could not adhere to dual antiplatelet treatment, or were allergic to arsenic trioxide, stainless steel alloy, polylactic acid polymer or contrast agents. Patients with any of the following forms of CHD: severe tortuosity, calcified lesions, two or more chronic proximal total occlusions; or in which a double stenting style was required to be 
performed for bifurcation lesions, were also excluded. The current study was completed in accordance with the Declaration of Helsinki and conducted with approval from the Ethics Committee of Chongqing Medical University. Each patient was informed of the nature of the study and had agreed to cooperate with postoperative follow-up and signed a form granting written informed consent.

Perioperative medication. Patients undergoing planned PCI were prescribed at least $100 \mathrm{mg}$ aspirin (Bayer AG, Leverkusen, Germany) and $75 \mathrm{mg}$ clopidogrel (Sanofi China, Hangzhou, China) orally each day for the three days prior to the procedure. Eight emergency patients who underwent PCI immediately chewed $300 \mathrm{mg}$ aspirin and 300-600 mg clopidogrel. Unfractionated $100 \mathrm{U} / \mathrm{kg}$ heparin (Tianjin Biochemical Pharmaceutical Co., Ltd, Tianjin, China) was injected intravenously during surgery. If the surgery lasted $>1 \mathrm{hr}$, an additional 1,000-2,000 $\mathrm{U}$ heparin was administered. For 5-7 days following surgery, 5,000 U/12 h low molecular weight heparin (Qilu Pharmaceuticals Co., Ltd, Jinan, China) was administered via subcutaneous injection. Aspirin was continued at $100 \mathrm{mg}$ per day throughout the remainder of the patients' lifetimes. Clopidogrel, at $75 \mathrm{mg}$ per day, was continued for $\geq 12$ months. Comprehensive treatments including lipid adjustment, blood pressure and blood glucose management were administered according to the patient's condition.

Interventional treatment. The Seldinger technique (16) was used to perform femoral artery or radial artery puncture. Coronary angiography was performed using the Judkins method (17) and stenting surgery was performed once the presence of lesions suitable for PCI was confirmed by coronary angiography. When multiple stents were necessary in long lesions, an overlap of 2-3 mm was required between stents and high-pressure balloon dilation was performed to secure the overlapping. When the implanted stent length was $\geq 29 \mathrm{~mm}$, high-pressure short balloon dilation to the same diameter as the diameter of the stent was performed once the stent was released. When residual stenosis of the lesion was $>20 \%$, a short balloon was used for dilation. The diameter ratio of the stent and the vasculature at the proximal and distal ends of the target lesion was 1:1.0-1.1, and the stents were placed to completely cover the lesion. Immediate success was indicated by a target lesion residual stenosis of $<20 \%$ and forward flow of thrombolysis in myocardial infarction (TIMI) level three (18), without PCI complications. These included main branch occlusion or pressure, thrombosis, huge hematoma, severe dissection or shock.

Follow-up indicators. The occurrence of major adverse cardiovascular events (MACE), including cardiac death, target lesion revascularization and nonfatal myocardial infarction, during hospitalization was documented. Regular cardiology clinic review and telephone follow-up were completed following patient discharge, in which the patient's medication, MACE occurrence and angina recurrence were recorded. Coronary angiography review was performed one year later, through which stent restenosis and thrombosis were recorded.

\section{Results}

Patient clinical characteristics. The selected 40 patients included 22 patients with acute myocardial infarction, 16 patients with unstable angina, one patient with stable angina and one patient with old myocardial infarction. Diabetes, hypertension and hyperlipidemia prevalence rates were $15.0,37.5$ and $17.5 \%$, respectively. The proportion of patients with a history of myocardial infarction, a family history of coronary heart disease or a history of smoking were $2.5,10.0$ and $80.0 \%$, respectively. No patients had previously undergone PCI.

Coronary angiography and stenting. Coronary angiography indicated that there were 20 cases of simple anterior descending artery disease, six cases of right coronary artery disease, four cases of circumflex artery lesions, eight cases of double vessel disease and two cases of triple vessel lesions and no cases of left main artery disease. A total of 63 stents were implanted into 52 AVI target lesions. The stent length was $23.4 \pm 7.2 \mathrm{~mm}$ and its diameter was $3.02 \pm 0.24 \mathrm{~mm}$. The immediate surgery success rate was $100 \%$. No MACE occurred during hospitalization.

Follow-up. All 40 patients completed the one-year clinical follow-up, meaning that the total follow-up rate was $100 \%$. During follow-up, one patient experienced gastrointestinal bleeding in the first two months following PCI, for which aspirin was discontinued and oral clopidogrel alone was administered for antiplatelet therapy; this patient experienced no recurrence of angina or MACE. The remaining 39 patients adhered to the aspirin and clopidogrel dual antiplatelet therapy for one year. The follow-up data concluded that the MACE rate was $15.0 \%(6 / 40)$ and the target lesion revascularization rate was $15.0 \%$ (6/40). Furthermore, no cardiac deaths or nonfatal myocardial infarctions occurred, the in-stent thrombosis rate was 0 and the angina recurrence rate was $32.5 \%$ (13/40). All patients agreed to undergo coronary arteriography review one year following surgery. Angiography revealed that the in-stent restenosis rate was $20.0 \%(8 / 40)$.

\section{Discussion}

The clinical application of drug-eluting stents (DES) is regarded as the third milestone in the development of PCI (19). Currently, the forms of DES that are widely used clinically include the sirolimus- and paclitaxel-eluting stents. As part of the Drug-eluting Stents project of Chinese Original products, the arsenic trioxide-eluting stent (AVI) has received eight utility model patents in China, the US, the EU, Japan and other countries, and was the first non-rapamycin/paclitaxel drug-eluting stent manufactured in China (20).

Although AVI has been used in the domestic market, only pre-marketing research data is currently available. Experimental results using New Zealand rabbits as animal models (11) indicated that no significant difference existed between $40 \mu \mathrm{g} \mathrm{As}_{2} \mathrm{O}_{3}$ DES and $180 \mu \mathrm{g}$ paclitaxel DES in reducing intimal hyperplasia. Experimental results using pigs as an animal model (14) demonstrated that AVI can 
reduce intimal proliferation following coronary artery damage without significantly delaying the vascular endothelial process at the stent implantation site and has a significant effect on restenosis rates. The results of this previous study demonstrated that after a 4-week follow-up period, the effect of $\mathrm{As}_{2} \mathrm{O}_{3}$ DES on restenosis rates was better than that of the stents without drug coating (14). Prior to the approval of AVI for use in PCI, randomized controlled trial data of AVI and sirolimus-eluting stents (20) demonstrated that out of 212 cases enrolled, who were randomly divided into groups receiving either arsenic trioxide $(n=106)$ or rapamycin $(n=106)$, the failure rate for the target vessels in the arsenic trioxide and rapamycin groups were 6.67 and $5.83 \%$ respectively $(\mathrm{P}=0.980)$ and the cardiac death or myocardial infarction event rate of the two groups were 0 and $3.88 \%$, respectively $(\mathrm{P}=0.058)$ following the two-year follow-up period. Target lesion revascularization rates were 6.67 and $1.94 \%$ respectively $(\mathrm{P}=0.170)$ and the identified stent thrombosis rate were 0 and $0.97 \%$, respectively $(\mathrm{P}=0.495)$. The angina recurrence rates of the two groups were 40.78 and $38.54 \%$, respectively $(\mathrm{P}=0.747)$. The nine month follow-up coronary angiography demonstrated that the in-stent restenosis rates of the two groups were 7.92 and $1.61 \%$ respectively $(\mathrm{P}=0.155)$, and the late lumen loss of the two stent groups were $0.29-0.52 \mathrm{~mm}$ and $0.10-0.25 \mathrm{~mm}$ respectively $(\mathrm{P}=0.008)$, indicating that the late lumen loss of the arsenic trioxide group was greater than that of the SES group (20).

The current study is a prospective clinical study for a monotherapy group, following the clinical approval of AVI, aimed at investigating the post-marketing clinical efficacy of AVI. The 40 cases selected were followed up for one year. The results indicate that the MACE rate was $15.0 \%$ (6/40), the target lesion revascularization rate was $15.0 \%(6 / 40)$, no cardiac death or nonfatal myocardial infarction events occurred, the occurrence of stent thrombosis rate was 0 and the angina recurrence rate was $32.5 \%$ (13/40). The one-year postoperative review of coronary angiography indicated that the in-stent restenosis rate was $20.0 \%(8 / 40)$. Therefore, the restenosis rate of the implanted AVI was higher, and its safety and clinical efficacy were lower than expected. In the light of the small sample size in this study, the short follow-up time, and the fact that it is a single-center clinical study, further studies are required to confirm these conclusions.

\section{References}

1. Chang H, Wang Q, Shi T, Huo K, Li C, Zhang Q, Wang G, Wang Y, Tang B, Wang W, et al: Effect of DanQi Pill on PPAR $\alpha$, lipid disorders and arachidonic acid pathway in rat model of coronary heart disease. BMC Complement Altern Med 16: 103, 2016.

2. Lee VW, Cheng FW, Choi AY, Fong ST, Yu CM and Yan BP: Clinical, humanistic, and economic outcomes between drug-eluting stent (DES) and bare metal stent (BMS): 18-month follow-up study. J Med Econ: 1-7, 31 Oct, 2016 (Epub ahead of print).
3. Babapulle MN and Eisenberg MJ: Coated stents for the prevention of restenosis: Part I. Circulation 106: 2734-2740, 2002.

4. Fricker J: Drug-eluting stents: Flashy future or flash-in-the-pan? Drug Discov Today 6: 1135-1137, 2001.

5. Regar E, Sianos G and Serruys PW: Stent development and local drug delivery. Br Med Bull 59: 227-248, 2001.

6. Dilda PJ and Hogg PJ: Arsenical-based cancer drugs. Cancer Treat Rev 33: 542-564, 2007.

7. Chen GQ, Shi XG, Tang W, Xiong SM, Zhu J, Cai X, Han ZG, $\mathrm{Ni}$ JH, Shi GY, Jia PM, et al: Use of arsenic trioxide (As2O3) in the treatment of acute promyelocytic leukemia (APL): I. As2O3 exerts dose-dependent dual effects on APL cells. Blood 89: 3345-3353, 1997.

8. Shen ZX, Shi ZZ, Fang J, Gu BW, Li JM, Zhu YM, Shi JY, Zheng PZ, Yan H, Liu YF, et al: All-trans retinoic acid/As2O3 combination yields a high quality remission and survival in newly diagnosed acute promyelocytic leukemia. Proc Natl Acad Sci USA 101: 5328-5335, 2004.

9. Lo-Coco F, Avvisati G, Vignetti M, Thiede C, Orlando SM, Iacobelli S, Ferrara F, Fazi P, Cicconi L, Di Bona E, et al: Retinoic acid and arsenic trioxide for acute promyelocytic leukemia. N Engl J Med 369: 111-121, 2013.

10. Zhang X, Yang XR, Sun C, Hu B, Sun YF, Huang XW, Wang Z, He YF, Zeng HY, Qiu SJ, et al: Promyelocytic leukemia protein induces arsenic trioxide resistance through regulation of aldehyde dehydrogenase 3 family member A1 in hepatocellular carcinoma. Cancer Lett 366: 112-122, 2015.

11. Hoffman EA, Gizelska K, Mirowski M and Mielicki W: Arsenic trioxide downregulates cancer procoagulant activity in MCF-7 and WM-115 cell lines in vitro. Contemp Oncol (Pozn) 19: 108-112, 2015.

12. Liu ZM, Tseng HY, Cheng YL, Yeh BW, Wu WJ and Huang HS: TG-interacting factor transcriptionally induced by $\mathrm{AKT} / \mathrm{FOXO} \mathrm{A}$ is a negative regulator that antagonizes arsenic trioxide-induced cancer cell apoptosis. Toxicol Appl Pharmacol 285: 41-50, 2015

13. Yang W, Ge J, Liu H, Zhao K, Liu X, Qu X, Li W, Huang Y, Sun A and Zou Y: Arsenic trioxide eluting stent reduces neointima formation in a rabbit iliac artery injury model. Cardiovasc Res 72: 483-493, 2006.

14. Shen L, Gong F, Tian W, Li W, Zhang F, Qian J, Sun A, Zou Y, Yang W and Ge J: Anti-inflammatory effects of arsenic trioxide eluting stents in a porcine coronary model. Biomed Res Int 2013: 937936, 2013

15. Li JX, Shen YQ, Cai BZ, Zhao J, Bai X, Lu YJ and Li XQ: Arsenic trioxide induces the apoptosis in vascular smooth muscle cells via increasing intracellular calcium and ROS formation. Mol Biol Rep 37: 1569-1576, 2010.

16. Seldinger SI: Catheter replacement of the needle in percutaneous arteriography. A new technique. Acta Radiol Suppl (Stockholm) 434: 47-52, 2008.

17. Li J, Elrashidi MY, Flammer AJ, Lennon RJ, Bell MR, Holmes DR, Bresnahan JF, Rihal CS, Lerman LO and Lerman A: Long-term outcomes of fractional flow reserve-guided vs. angiography-guided percutaneous coronary intervention in contemporary practice. Eur Heart J 34: 1375-1383, 2013.

18. The Thrombolysis in Myocardial Infarction (TIMI) trial. Phase I findings. TIMI Study Group. N Engl J Med 312: 932-936, 1985.

19. Byrne RA, Sarafoff N, Kastrati A and Schömig A: Drug-eluting stents in percutaneous coronary intervention: A benefit-risk assessment. Drug Saf 32: 749-770, 2009.

20. Shen L, Yang W, Yin JS, Liu XB, Wu YZ, Sun AJ, Qian JY and Ge JB: Nine-month angiographic and two-year clinical follow-up of novel biodegradable-polymer arsenic trioxide-elutin gstent versus durable-polymer sirolimus-eluting stent for coronary artery disease. Chin Med J (Engl) 128: 768-773, 2015. 